Comparación del costo y del costo-efectividad de los mosquiteros tratados con insecticida y de la fumigación domiciliaria residual en Sudáfrica

La fumigación intradomiciliaria residual (FIDR) ha sido la principal medida de prevención de la malaria en Sudáfrica durante más de 50 años y se ha considerado que, junto con la detección activa y pasiva de casos, ha sido muy eficaz en la región de KwaZulu-Natal. Sin embargo, en los últimos años se han señalado varios puntos débiles de la FIDR que podrían ser abordados cambiándola por el uso de mosquiteros tratados con insecticida (MTI), cuya eficacia ha sido demostrada en otras regiones de África y que, además, podrían ser más baratos. En África solo se ha hecho una comparación directa entre la FIDR y los MTI en Tanzania, comprobándose que la eficacia era similar, pero que los MTI eran significativamente más baratos. Sin embargo, la situación en Sudáfrica es muy diferente en aspectos como la transmisión de la malaria, la organización de la lucha contra la enfermedad y el entorno socioeconómico.

Con el fin de proporcionar datos que fundamenten la elección de la FIDR o de los MTI en Sudáfrica, el Consejo Sudafricano de Investigaciones Médicas y el Departamento de Salud organizaron en 1998-1999 un ensayo clínico en el norte de KwaZulu-Natal para comparar la práctica actual de la FIDR con deltametrina (una fumigación al año) y el uso de MTI tratados con deltametrina o permetrina (un tratamiento al año). En este artículo se describen los resultados de la comparación de costos y se combinan estos datos con los resultados de la eficacia para calcular la relación costo-efectividad de los MTI frente a la FIDR.

En la zona estudiada, rural y de población dispersa (aproximadamente 32000 habitantes) la transmisión de la malaria es endémica, pero baja, con una incidencia anual de casos confirmados cercana al 5\%; todos los grupos de edad son vulnerables. Antes de este estudio, nunca se habían utilizado MTI en la zona.

Se identificaron siete pares de zonas de casas rurales dispersas, apareadas en función de la incidencia de malaria. Aleatoriamente, una zona de cada par fue asignada a la FIDR y la otra a los MTI. La población total de las 14 zonas era de 13099 habitantes en 1996. En 1996 se fumigaron todas las zonas; en 1997 se volvieron a fumigar todas las zonas, pero se distribuyeron MTI en las asignadas a este grupo. En 1998 y 1999 ya no se realizaron FIDR en las zonas asignadas al grupo MTI. La FIDR tuvo lugar cada año, entre septiembre y diciembre, antes de la temporada de malaria; la dosis fue de $20 \mathrm{mg} / \mathrm{m}^{2}$. Se realizaron campañas de información acerca del uso de los MTI, que fueron tratados con una dosis de $200 \mathrm{mg} / \mathrm{m}^{2}$.

La principal medida de eficacia fue el número de casos confirmados de parasitemia, registrados prospectivamente mediante detección activa y pasiva. El número de casos evitados por los MTI, o sea, el número de casos que hubieran ocurrido en las zonas asignadas a los MTI si hubieran sido cubiertas con FIDR, se calculó multiplicando las personas-año de las zonas MTI por la incidencia media en 1998 y 1999, dividido por la razón de riesgo ajustada. El tamaño de la muestra no fue suficiente para evaluar la diferencia de mortalidad entre las dos intervenciones. Sin embargo, utilizando la tasa de letalidad de la región en 1998 y 1999, fue posible estimar las muertes evitadas, extrapoladas de los casos evitados. La medición de la eficacia se limitó al impacto sobre el número de casos en las zonas cubiertas por la intervención, pero no fue posible cuantificar los beneficios adicionales, derivados de la reducción de la diseminación de la transmisión a otras zonas de la provincia, a pesar de que la creación de esta barrera se considera un aspecto clave del control eficaz.

En los cálculos de los costos se incluyeron todas las actividades de FIDR y MTI, pero no los costos de otras actividades de control (larvicidio, detección pasiva y activa de casos, bioensayos, etc.), ni tampoco los gastos de gestión, pues se consideró que serían similares con ambas intervenciones. Los costos fueron considerados desde la perspectiva del proveedor; las familias no tuvieron gastos personales relacionados con las intervenciones. Todos los costos fueron convertidos en rands sudafricanos (ZAR) de 1999 utilizando el índice de precios al consumo, y en dólares estadounidenses (USD) utilizando el cambio de enero del 2000: USD1 = ZAR6,12. Con respecto a la FIDR, se contabilizaron los costos que contribuyeron a los resultados de eficacia de 1998 y 1999, esto es, las FIDR de 1997 y 1998, respectivamente. Los costos de los MTI se desglosaron en costos de desarrollo y costos recurrentes. Se calcularon los costos tanto económicos como financieros de ambas intervenciones. 
Los MTI fueron significativamente más eficaces que las FIDR en la reducción de los casos de malaria. Las razones de probabilidades ajustadas fueron de 0,67 en 1998 (intervalo de confianza [IC]: 0,61 a 0,72 ) y 0,72 en 1999 (IC: 0,65 a 0,79). Combinando los datos de ambos años, la cifra fue de 0,69 (IC: 0,65 a 0,73). En los 2 años, los MTI evitaron 855 casos de malaria (520 en 1998 y 335 en 1999) y 8 muertes.

Con los MTI, el 74\% de los costos financieros correspondieron a la fase de desarrollo (ZAR61,89 por persona). Los costos recurrentes fueron mucho menores (ZAR8,99 en 1998 y ZAR9,25 en 1999). El costo por persona de la FIDR fue similar en 1998 (ZAR15,88) y 1999 (ZAR15,19). Para la FIDR, la diferencia entre los costos económico y financiero fue pequeña, pero para los MTI fue más importante. El costo económico por persona cubierta a lo largo de los 2 años fue de ZAR29,22 para la FIDR y ZAR46,59 para los MTI.

Aplicando un costo de fármacos de ZAR12,68 por persona a los 855 casos evitados por los MTI, el ahorro fue de ZAR10 835 (ZAR1,99 per cápita).

El cálculo de la razón costo-efectividad incremental bruta reveló que el uso de MTI en vez de la FIDR costaba ZAR111 más (USD18) por caso adicional evitado y ZAR11 718 (USD1 915) por muerte evitada. Incluyendo el ahorro en fármacos por caso evitado, las cifras correspondientes fueron de ZAR98 (USD16) y ZAR10 377 (USD1 696), respectivamente.

En un análisis de sensibilidad, el mayor costo económico de los MTI en comparación con la FIDR fue bastante estable a las variaciones de los diferentes parámetros. No obstante, se comprobó que el costo era bastante sensible a los cambios del precio y de la vida media de los mosquiteros, así como al precio del insecticida, y que el cambio a los MTI podría proporcionar ahorros financieros netos si el precio de los mosquiteros bajara por debajo de USD3,57 (ZAR21,85) o si un cambio de política permitiera reducir de forma importante el personal permanente y a tiempo completo del programa de lucha contra la malaria.

Estos resultados revelan que los MTI son significativamente más eficaces que la FIDR para evitar casos de malaria, pero también más caros. Teniendo en cuenta su mayor eficacia, los políticos podrían considerar que los MTI constituyen un uso de los recursos con una buena relación costoefectividad, aunque los costos económicos sean elevados. (Goodman, Mnzava, Dlamini, Sharp, Mthembu, Gumede. Comparison of the cost and cost-effectiveness of insecticide-treated bednets and residual house-spraying in KwaZulu-Natal, South Africa. Trop Med Intl Health 2001;6:280-295)

\section{Prevención de la diabetes de tipo 2 mediante cambios del estilo de vida en individuos con intolerancia a la glucosa}

La diabetes de tipo 2 es el resultado de la interacción entre una predisposición genética y factores de riesgo conductuales y ambientales, entre los que destacan la obesidad y el sedentarismo. La intolerancia a la glucosa representa un estado intermedio entre la tolerancia normal a la glucosa y la diabetes manifiesta. Los individuos con intolerancia a la glucosa corren mayor riesgo de padecer diabetes de tipo 2 y constituyen, por lo tanto, un importante grupo destinatario de intervenciones para prevenir la diabetes. El Estudio Finlandés de Prevención de la Diabetes se realizó con el fin de determinar la factibilidad y los efectos de un programa de cambio del estilo de vida destinado a prevenir o retrasar la aparición de diabetes de tipo 2 en individuos con intolerancia a la glucosa.

Se consideraron elegibles para participar en el estudio las personas con sobrepeso (índice de masa corporal $\geq 25$ ), de 40 a 65 años, con intolerancia a la glucosa (glucemia de 140 a $200 \mathrm{mg} / \mathrm{dL} 2$ horas después de la administración oral de $75 \mathrm{~g}$ de glucosa cuando la glucemia en ayunas era $<140 \mathrm{mg} / \mathrm{dL}$ ). Se excluyeron aquellos con diabetes sacarina, enfermedades crónicas que hicieran improbable la supervivencia a los 6 años y características físicas o psicológicas que pudieran obstaculizar su participación en el estudio.

Los 522 participantes ( 172 hombres y $350 \mathrm{mu}$ jeres) fueron asignados aleatoriamente al grupo que recibió la intervención (grupo activo) o al grupo de control. La asignación fue estratificada en función del centro, del sexo y de la glucemia 2 horas después de la sobrecarga de glucosa (140 a 169 o 170 a $200 \mathrm{mg} / \mathrm{dL}$ ). El estudio solo fue parcialmente enmascarado. La intervención consistió en asesoramiento individualizado destinado a conseguir una reducción del peso y de la ingesta de grasas totales y saturadas, y un aumento de la ingesta de fibra y de la actividad física. A los individuos del grupo de control se les proporcionó información general, oral y escrita, acerca de la dieta y el ejercicio en la consulta inicial y en las consultas anuales, pero no recibieron asesoramiento específico individualizado. La consulta inicial y las consultas anuales incluyeron la anamnesis y exploración física, mediciones antropométricas y de la tensión arterial, prueba de tolerancia a la glucosa oral y determinaciones de la insulina sérica, colesterol total, colesterol de las lipoproteínas de alta densidad y triglicéridos.

La diabetes se definió según los criterios de 1985 de la Organización Mundial de la Salud: glucemia en ayunas $\geq 140 \mathrm{mg} / \mathrm{dL}$ o glucemia $\geq 200$ 
mg/dL 2 horas después de una sobrecarga de glucosa. Para establecer el diagnóstico de diabetes se requirió su confirmación por una segunda prueba de tolerancia a la glucosa oral. Si esta segunda prueba no confirmó los resultados de la anterior, el participante permaneció en el grupo al que había sido asignado inicialmente. Todos los análisis se basaron en el principio de la intención de tratar.

El primer participante fue asignado en noviembre de 1993 y el último en junio de 1998; 90\% de los individuos han participado en el estudio durante al menos 2 años. La duración media del período de observación es de 3,2 años. Las características basales de los dos grupos eran similares. Durante el primer año, el peso corporal medio ( \pm desviación típica) disminuyó en $4,2 \pm 5,1 \mathrm{~kg}$ $(4,7 \pm 5,4 \%)$ en el grupo activo y en $0,8 \pm 3,7 \mathrm{~kg}$ $(0,9 \pm 4,2 \%)$ en el grupo de control $(P<0,001)$. El perímetro de la cintura, la glucemia en ayunas y la glucemia y la insulinemia 2 horas después de la sobrecarga de glucosa presentaron una reducción significativamente mayor en el grupo activo que en el de control. A los 2 años, las disminuciones del peso, de la glucemia en ayunas y de la glucemia 2 horas después de la sobrecarga de glucosa seguían siendo significativamente mayores en el grupo activo que en el de control. Además, el grupo activo también presentó disminuciones significativamente mayores de la insulinemia 2 horas después de la sobrecarga de glucosa, de la concentración de triglicéridos y de la tensión arterial.

El éxito en la consecución de los objetivos de la intervención, calculado a partir de los diarios sobre la dieta y de los cuestionarios sobre el ejercicio, osciló entre el $25 \%$ para la ingesta de fibra y el $86 \%$ para el ejercicio.

Se diagnosticó diabetes en 86 individuos: 27 del grupo activo y 59 del grupo de control. La proporción media de individuos en los que la intolerancia a la glucosa progresó hacia la diabetes fue del 3\% al año en el grupo activo y del $6 \%$ en el grupo de control. La incidencia absoluta de diabetes fue de 32 y 78 casos por 1000 personas-año en uno y otro grupo, respectivamente.

La incidencia acumulada de diabetes fue menor en el grupo activo que en el de control. La diferencia fue estadísticamente significativa a los 2 años: $6 \%$ en el grupo activo (intervalo de confianza del $95 \%$ [IC95\%]: 3 a 9\%) y $14 \%$ en el grupo de control (IC95\%: 10 a 19\%). A los 4 años, las cifras correspondientes fueron del 11\% (IC95\%: 6 a 15\%) y 23\% (IC95\%: 17 a 29\%). Mediante análisis de regresión de Cox se estimó que la incidencia acumulada de diabetes fue un 58\% menor en el grupo activo que en el de control (razón de riesgo instantáneo [hazard ratio]: 0,4; IC95\%: 0,3 a 0,7; $P<0,001$ ): 63\% para los hombres (IC95\%: 18 a $79 \% ; P=0,01$ ) y $54 \%$ para las mujeres (IC95\%: 26 a 81\%; $P=0,008$ ).

Los autores concluyen que este estudio aporta pruebas de que es posible prevenir la diabetes de tipo 2 mediante cambios del estilo de vida de los individuos que se encuentran en riesgo de padecer la enfermedad. A pesar de que la pérdida de peso fue pequeña en el grupo activo, la diferencia entre los dos grupos con respecto a la incidencia de diabetes fue considerable, lo cual demuestra la importancia de la pérdida de peso, aunque sea pequeña, en la prevención de esta enfermedad. Por otra parte, la consecución del modesto objetivo de realizar al menos 4 horas de ejercicio a la semana también se asoció a una significativa reducción del riesgo de diabetes en los individuos que no perdieron peso. Además, en este estudio, los cambios del estilo de vida también redujeron otros factores de riesgo cardiovascular, como los triglicéridos y la tensión arterial. Aunque a menudo se afirma que es difícil cambiar el estilo de vida, este estudio indica que ese pesimismo no parece estar justificado.

En resumen, los resultados obtenidos indican que es posible conseguir una prevención primaria de la diabetes de tipo 2 mediante intervenciones no farmacológicas que se pueden poner en práctica en el ámbito de la atención primaria; para evitar un caso de diabetes, sería necesario tratar de esta forma a 22 individuos con intolerancia a la glucosa durante un año, o a 5 individuos durante 5 años. (Tuomilehto J, Lindstrom J, Eriksson JG, Valle TT, Hamalainen $\mathrm{H}$, Ilanne-Parikka $\mathrm{P}$, et al. Prevention of type 2 diabetes mellitus by changes in lifestyle among subjects with impaired glucose tolerance. $\mathrm{N}$ Engl J Med 2001;344:1343-1350.)

\section{Efecto de los preservativos sobre la reducción de la transmisión del virus del herpes simple de tipo 2 del hombre a la mujer}

La infección por el virus del herpes simple de tipo 2 (VHS-2) es una de las enfermedades de transmisión sexual más frecuentes en los Estados Unidos de América, donde se estima que cerca de un 22\% de los mayores de 12 años están infectados. La gran mayoría de estas infecciones son adquiridas por contacto sexual. Como no existen estudios prospectivos acerca de la capacidad de los preservativos para reducir la transmisión del virus, los autores de este estudio se propusieron investigar los factores de riesgo de adquisición del VHS-2 y la eficacia de los preservativos para reducir su transmisión.

Con este objetivo, se analizaron los datos de un ensayo clínico aleatorizado, doblemente enmascarado y controlado con placebo, de 18 meses de 
duración, en el que se investigó la eficacia de una posible vacuna frente al VHS-2.

Los participantes fueron individuos sanos, seronegativos para el VHS-2 y el virus de la inmunodeficiencia humana (VIH), que tenían relaciones monogámicas durante al menos 6 meses con una persona seropositiva para el VHS-2 y padecían herpes genital recurrente. Los miembros susceptibles de la pareja tuvieron 11 consultas programadas a lo largo de los 18 meses y rellenaron un diario en el que registraron sus contactos sexuales con la pareja o con otras personas. La información recogida incluyó el número de relaciones sexuales (vaginales o anales), el uso de preservativos, el número de contactos en los que la pareja tenía lesiones herpéticas, el uso de aciclovir por la pareja y el número de nuevos contactos sexuales. El uso diario crónico de tratamiento antivírico por la pareja infectada fue motivo de exclusión del estudio (18 casos; 3,4\%), aunque estos casos se incluyeron en el análisis.

Como medida primaria de eficacia se utilizó la adquisición de infección genital por VHS-2 por parte del miembro susceptible de la pareja. Estos casos se compararon con aquellos que se mantuvieron sin infección con respecto a sus características demográficas, a la actividad sexual y al uso de preservativos.

En el estudio participaron 528 parejas monogámicas. Entre los miembros susceptibles de estas parejas, en su mayoría (98\%) heterosexuales, había 261 hombres y 267 mujeres, con una edad mediana de 36 años; el $41 \%$ era seronegativo para el VHS y el $59 \%$ era seropositivo para el VHS-1. En el examen inicial, la frecuencia mediana de relaciones sexuales fue de dos a la semana (similar en ambos sexos) y la mitad de los casos solo utilizaban preservativos en $\leq 10 \%$ de las ocasiones.

Durante el período de observación, 31 individuos $(5,9 \%)$ adquirieron infección por el VHS-2: 26 mujeres $(9,7 \%)$ y 5 hombres $(1,9 \%)$. La tasa de adquisición de la infección por acto sexual fue cerca de seis veces mayor en las mujeres que en los hombres: 8,9 frente a 1,5 por 10000 actos sexuales, respectivamente.

Los factores de riesgo de adquisición de la infección por VHS-2 se investigaron con modelos univariados y multivariados. En los análisis univariados, los factores relacionados de forma significativa con el riesgo fueron el sexo (mayor riesgo en el sexo femenino), la edad (mayor riesgo en los individuos más jóvenes), la raza (mayor riesgo en los no blancos), el estado serológico de la pareja infectada (mayor riesgo en los infectados tanto por el VHS-1 como por el VHS-2) y la frecuencia de las relaciones sexuales (mayor riesgo a mayor frecuencia). La mayor frecuencia de las recurrencias del herpes genital en el miembro infectado de la pareja, el uso menos frecuente de preservativos y la seronegatividad frente a los VHS del miembro susceptible de la pareja también se asociaron al riesgo, pero de forma no significativa. En el análisis multivariado estratificado por sexos, los factores asociados de forma estadísticamente significativa a un mayor riesgo fueron los siguientes: edad más joven (razón de riesgo instantáneo [hazard ratio: HR] ajustada, por cada 5 años: 1,57; IC95\%: 1,22 a 2,04), seropositividad para el VHS-1 y el VHS-2, frente a seropositividad únicamente para el VHS-2 (HR ajustada: 2,34; IC95\%: $1,14$ a 4,82$)$ y mayor frecuencia de las relaciones sexuales (HR ajustada, por cada acto sexual adicional a la semana: 1,10; IC95\%: 1,01 a 1,19). Además, la mayor frecuencia del uso de preservativos se relacionó con una menor adquisición de la infección (HR: 0,08; IC95\%: 0,01 a 0,60) en los participantes que los usaban en más del $50 \%$ de los actos sexuales, en comparación con los que los usaban con menor frecuencia. El uso de preservativos en más del $25 \%$ de los actos sexuales también se asoció a una protección frente a la adquisición de la infección por VHS-2 en las mujeres (HR ajustada: 0,085; IC95\%: 0,01 a 0,67 ), pero no en los hombres (HR ajustada: 2,02; IC95\%: 0,32 a 12,50).

En este estudio, la tasa de transmisión del VHS-2 del hombre a la mujer $(8,9 / 10000$ actos sexuales) fue similar a la tasa de adquisición del VIH por vía sexual. Uno de sus resultados más importantes fue que demostrara por primera vez que el riesgo de transmisión está relacionado con los comportamientos sexuales, aumentando a medida que lo hace la actividad sexual y disminuyendo con el uso de preservativos, al menos en las mujeres susceptibles. Con los datos obtenidos, se puede calcular que el uso de preservativos en más del $25 \%$ de las relaciones sexuales permitiría reducir en 315000 el número anual de nuevos casos de VHS-2 en mujeres.

La población estudiada presenta importantes diferencias con respecto a la mayoría de las parejas discordantes para el VHS-2, primero porque ambos miembros de la pareja sabían que uno de ellos estaba infectado, y segundo porque tenían relaciones monogámicas hacía, como mínimo, 6 meses. (Wald A, Langenberg AGM, Link K, Izu AE, Ashley R, Warren $\mathrm{T}$, et al. Effect of condoms on reducing the transmission of Herpes simplex virus type 2 from men to women. JAMA 2001;285: 3100-3106)

\section{Nueva estrategia eficaz de lucha contra la malaria en lactantes africanos}

En zonas donde la transmisión de Plasmodium falciparum es alta, como en el sur de Tanzania, cerca 
de la mitad de los ingresos hospitalarios y de las muertes por malaria ocurren en menores de 1 año. Como los síntomas y signos de la anemia son inespecíficos, no es raro que la enfermedad no sea diagnosticada y tratada. Además, el tratamiento preventivo, en vez de curativo, podría reducir el impacto de la falta de acceso a servicios curativos.

En zonas endémicas, se ha demostrado que la quimioprofilaxis antimalárica reduce la morbilidad por malaria, el absentismo escolar y la mortalidad por todas las causas. Aunque se ha utilizado en algunos niños africanos en edad escolar, la quimioprofilaxis se ha abandonado debido al temor a la aparición de resistencia y a la pérdida o retraso de la adquisición de la inmunidad adquirida.

Con el fin de potenciar al máximo el efecto protector de la quimioprofilaxis sin poner en peligro el desarrollo de la inmunidad frente a la malaria, los autores de este estudio investigaron el empleo del tratamiento antimalárico intermitente en el primer año de vida, independientemente de la presencia de parásitos o de síntomas.

Para ello se realizó un estudio aleatorizado, doblemente enmascarado y controlado con placebo en niños tanzanos que recibieron dosis intermitentes de sulfadoxina-pirimetamina junto con las vacunaciones del Programa Ampliado de Vacunaciones de la Organización Mundial de la Salud.

El estudio se llevó a cabo en Ifakara, una zona semirrural del sur de Tanzania, con una población estimada de 55000 habitantes en la que los lactantes representan un $44 \%$ de todos los ingresos pediátricos por malaria y un $54 \%$ de las muertes por esta causa en pacientes pediátricos ingresados. La sulfadoxina-pirimetamina es en la actualidad el tratamiento antimalárico de primera línea recomendado en Tanzania. El cumplimiento de las vacunaciones es elevado en la zona. El 92\% de los niños reciben las tres dosis de las vacunas contra la difteria, tétanos y tos ferina y de la vacuna antipoliomielítica oral (DTP/OPV), mientras que el $80 \%$ recibe la vacuna contra el sarampión. Los lactantes fueron reclutados entre agosto de 1999 y abril del 2000, inmediatamente después de recibir la segunda dosis de DTP/OPV (2 meses de edad). Las dosis de sulfadoxina-pirimetamina o placebo fueron ajustadas en función del peso corporal: un cuarto de comprimido en los de peso $<5 \mathrm{~kg}$, medio comprimido en los de $5-10 \mathrm{~kg}$ y un comprimido en los de $>10 \mathrm{~kg}$. La segunda dosis se administró inmediatamente después de la tercera dosis de DTP/OPV (3 meses), y la tercera en el momento de la vacunación contra el sarampión (9 meses). Todos los niños recibieron suplementos de hierro entre los 2 y los 6 meses de edad. En cada consulta se realizó un detallado cuestionario sobre los signos y síntomas y se obtuvieron muestras de sangre para investigar la presencia de plasmodios. El examen de la parasitemia se repitió nuevamente a los 12 y a los 18 meses de edad.

Las variables primarias de eficacia fueron el primer o único episodio clínico de malaria y la anemia grave entre el momento del reclutamiento y el año de edad. Los episodios clínicos de malaria se definieron como la presencia de una temperatura axilar de al menos $37,5^{\circ} \mathrm{C}$, junto con cualquier densidad de parasitemia por P. falciparum asexuado. Esta definición tiene una sensibilidad del 100\% y una especificidad del $97 \%$.

De acuerdo con las estimaciones actuales de la frecuencia de la malaria y de la anemia en la zona, se calculó que sería necesaria una muestra de 700 niños para obtener una potencia del $80 \%$ para detectar una reducción de estas variables en un $30 \%$. El análisis primario de eficacia se basó en la intención de tratar. El riesgo de sufrir un primer episodio o un único episodio de malaria entre el momento del reclutamiento y los 12 meses de edad se calculó con modelos de regresión de Cox. En el análisis secundario solo se incluyeron los niños que habían recibido las tres dosis previstas de sulfadoxina-pirimetamina o placebo; el objetivo de este análisis consistió en valorar el efecto del incumplimiento del tratamiento sobre las estimaciones de eficacia obtenidas en el análisis primario.

De los 701 niños reclutados, 661 (94\%) completaron el estudio. Las causas de abandono fueron la muerte (16), la emigración (11), la revocación del consentimiento por parte de los padres (12) y otros motivos (1). Los grupos asignados de forma aleatoria al tratamiento activo o con placebo fueron similares con respecto a las características basales, a la proporción de casos que finalizaron el estudio, al número y tipo de abandonos y al cumplimiento del tratamiento con suplementos de hierro. Un total de 540 niños $(77 \%)$ recibieron las tres dosis de sulfadoxina-pirimetamina o placebo.

No se notificaron reacciones cutáneas graves. Aunque el número de muertes y de consultas externas fue similar en ambos grupos, los niños tratados con sulfadoxina-pirimetamina tuvieron un número significativamente menor de ingresos hospitalarios. Las únicas diferencias significativas asociadas al tratamiento con sulfadoxina-pirimetamina consistieron en un aumento del riesgo de consulta externa por otitis media (riesgo relativo [RR]: 1,9; intervalo de confianza del 95\% [IC95\%]: 1,2 a 3,0; $P=0,011$ ), una reducción del riesgo de presentar signos de infección de las vías respiratorias inferiores (RR: 0,7; IC95\%: 0,4 a 1,0; $P=0,05$ ) y de presentar erupciones cutáneas por causas distintas de la escabiosis en el mes siguiente a la segunda dosis (RR: 0,2; IC95\%: $0,06$ a 0,$70 ; P=0,01)$. 
El tratamiento activo tuvo un importante efecto protector sobre el número de primeros o únicos episodios clínicos de malaria. La modificación de la definición de los casos de malaria y la adopción de diferentes métodos de análisis no cambió esta estimación. Hubo nueve episodios de malaria grave en el grupo tratado con placebo y cinco en el tratado con sulfadoxina-pirimetamina (eficacia protectora del 44\% [IC95\%: -68 a 81\%], $P=0,29)$. El tratamiento activo también redujo la tasa de anemia grave a la mitad. Los tres únicos casos de anemia potencialmente mortal ocurrieron en lactantes tratados con placebo. El análisis secundario limitado a los niños que recibieron las tres dosis de sulfadoxina-pirimetamina proporcionó una eficacia protectora del $61 \%$ (IC95\%: 40 a 75\%) con respecto a la malaria y del 56\% (IC95\%: 7 a 79\%) con respecto a la anemia grave. Las tasas de malaria y anemia grave no aumentaron tras la finalización del tratamiento con sulfadoxina-pirimetamina. Los índices antropométricos y la prevalencia de fiebre, parasitemia por $P$. falciparum sexuado y asexuado y anemia fueron similares a los 12 y 18 meses de edad.

De acuerdo con estos resultados, el tratamiento intermitente con sulfadoxina-pirimetamina fue seguro, bien tolerado y redujo la tasa de malaria clínica en un $59 \%$, la tasa de anemia grave en un $50 \%$, el número de ingresos hospitalarios en un $30 \%$ y la tasa de episodios febriles por cualquier causa en un $13 \%$. La disminución de la anemia en el grupo tratado con sulfadoxina-pirimetamina ocurrió a pesar de que todos los niños recibieron profilaxis con sulfato ferroso, cuya eficacia está demostrada. En opinión de los autores, estos resultados indican que el tratamiento intermitente con sulfadoxina-pirimetamina en lactantes probablemente tenga importantes repercusiones para la salud pública en zonas endémicas como la estudiada.

La prevalencia de la parasitemia en niños tratados con placebo fue baja ( $4 \%$ a los 12 meses); por consiguiente, los efectos protectores de la sulfadoxina-pirimetamina parecen estar más relacionados con una acción profiláctica que con la eliminación de los parásitos en los escasos niños que estaban parasitados al inicio del tratamiento y, siendo así, parece más adecuado el nombre de profilaxis intermitente que el de tratamiento intermitente.

No es de esperar que este tratamiento intermitente tenga grandes repercusiones sobre la aparición de resistencia, entre otros motivos porque el problema de las dosis subterapéuticas es mínimo, una vez que las tomas son observadas directamente por el personal sanitario.

En un estudio anterior realizado en la misma zona, la quimioprofilaxis continua entre los 2 y los 12 meses de edad se asoció a un notable aumento de la tasa de malaria clínica tras la interrupción de la intervención, hecho interpretado como un retraso o inhibición de la inmunidad específica frente a esta enfermedad. En el presente estudio no hubo ningún indicio de un efecto de rebote similar. Apenas existen datos acerca de los posibles efectos de las medicaciones antimaláricas sobre la respuesta serológica a las vacunaciones. En este aspecto, fue tranquilizante la ausencia de diferencias entre los dos grupos con respecto a la tasa de seroconversión. (Schellenberg D, Menendez C, Kahigwa E, Aponte J, Vidal J, Tanner M, et al. Intermittent treatment for malaria and anaemia control at time of routine vaccinations in Tanzanian infants: a randomised, placebo-controlled trial. Lancet 2001;357:1471-4177.)

\section{Eficacia de la vacuna contra la varicela en la práctica clínica}

En 1995 se aprobó en los Estados Unidos de América el uso de una vacuna de virus vivos atenuados contra la varicela en individuos de más de 12 meses de edad susceptibles a la enfermedad. Dicha aprobación se basó fundamentalmente en los resultados de un ensayo clínico, pero la eficacia de las vacunas en la práctica clínica puede ser muy diferente de la observada en ensayos clínicos. En este estudio de casos y controles se analiza la eficacia de la vacuna, tal como se usa actualmente en la práctica clínica en EE.UU.

Los participantes fueron niños sanos de 13 meses a 16 años de edad. Se excluyeron los niños inmunodeprimidos, en los que no se recomienda el empleo de la vacuna, y aquellos con antecedentes de varicela. Asimismo, se excluyeron los que habían recibido la vacuna en las 4 semanas anteriores, tiempo que tarda el individuo en crear anticuerpos tras la vacunación.

Los casos fueron los niños con sospecha de varicela atendidos en los 15 centros pediátricos participantes, identificados mediante vigilancia activa. La enfermedad se confirmó mediante la reacción en cadena de la polimerasa para detectar la presencia del ADN del virus en las lesiones. Por cada caso potencial de varicela se seleccionaron dos controles emparejados por edad ( \pm 1 mes) y centro médico de referencia.

Se calcularon las razones de los productos cruzados (odds ratios: OR) de la vacunación, sus intervalos de confianza del 95\% (IC95\%) y los valores $P$. La eficacia de la vacuna se calculó restando la OR a la unidad (1 - OR). Las estimaciones fueron ajustadas por los efectos de posibles factores de confusión mediante regresión logística condicional.

Entre marzo de 1997 y noviembre del 2000 se identificaron 461 niños con sospecha de varicela, de los cuales 118 fueron excluidos por diferentes moti- 
vos. Como controles participaron 500 niños. La reacción en cadena de la polimerasa fue positiva en 243 de los 330 posibles casos de varicela $(74 \%)$. La enfermedad fue menos grave en los niños que habían recibido la vacuna contra la varicela. De los 56 niños vacunados con varicela, el $86 \%$ tenía enfermedad leve, mientras que de los 187 no vacunados con varicela, solo el $48 \%$ tenía enfermedad leve $(P<0,001)$. Las estimaciones de la eficacia de la vacuna se basaron en los 202 de estos 243 niños en los que existían datos completos acerca de uno o ambos controles (total: 389 controles).

La probabilidad de haber recibido la vacuna era sustancialmente menor en los niños con varicela que en los controles (23\% frente a $61 \%$ ). La eficacia global de la vacuna fue del 85\% (IC95\%: 78 a 90\%; $P<0,001)$. Con respecto a la enfermedad grave o moderadamente grave, la eficacia fue del $97 \%$ (IC95\%: 93 a 99\%; $P<0,001$ ). Para los niños de menos de 5 años, la eficacia fue del 79\% (IC95\%: 61 a $89 \%$; $P<0,001)$; para los de 5 a 10 años, del $89 \%$ (IC95\%: 80 a $94 \% ; P<0,001)$, y para los mayores de 10 años, del 92\% (IC95\%: 45 a 99\%; $P=0,03$ ).

Estos resultados indican que, tal como se usa en la actualidad en la práctica clínica, la vacuna contra la varicela posee una eficacia excelente, al menos a corto plazo. (Vazquez M, LaRussa PS, Gershon AA, Steinberg SP, Freudigman K, Shapiro ED. The effectiveness of the varicella vaccine in clinical practice. N Engl J Med 2001;344:955-960)

\section{Eficacia y seguridad de la rehidratación oral con una solución de osmolaridad reducida en niños con diarrea acuosa aguda}

Durante 20 años, en los casos de deshidratación por diarrea aguda de cualquier origen, la Organización Mundial de la Salud (OMS) ha recomendado la rehidratación oral con una solución que contiene $90 \mathrm{mmol} / \mathrm{L}$ de sodio (Na) y $111 \mathrm{mmol} / \mathrm{L}$ de glucosa (osmolaridad total, $311 \mathrm{mosm} / \mathrm{L}$ ). La eficacia de este tratamiento está perfectamente establecida, pero sigue habiendo discrepancias acerca de la composición ideal de la solución de rehidratación oral (SRO). En este ensayo clínico multicéntrico, aleatorizado y doblemente enmascarado, se analiza la seguridad y eficacia de una SRO de osmolaridad reducida, en comparación con la SRO tradicionalmente recomendada por la OMS.

La SRO de osmolaridad reducida tuvo la siguiente composición: $75 \mathrm{mmol} / \mathrm{L}$ de sodio $(\mathrm{Na}), 20$ $\mathrm{mmol} / \mathrm{L}$ de potasio $(\mathrm{K}), 65 \mathrm{mmol} / \mathrm{L}$ de cloruro, 10 $\mathrm{mmol} / \mathrm{L}$ de citrato y $75 \mathrm{mmol} / \mathrm{L}$ de glucosa, con una osmolaridad de $245 \mathrm{mosm} / \mathrm{L}$.

El estudio se realizó en niños con diarrea aguda de cinco países en vías de desarrollo (Ban- gladesh, Brasil, India, Peru y Vietnam). La admisión de pacientes comenzó en junio de 1995 y terminó en febrero de 1997. Las medidas de eficacia analizadas fueron la defecación en las 24 horas posteriores a la asignación aleatoria, la proporción de niños que necesitaron tratamiento intravenoso no programado, la proporción de niños con vómitos en las primeras 24 horas y la duración de la diarrea tras la asignación aleatoria.

Los participantes fueron niños del sexo masculino (debido a la necesidad de separar las heces de la orina en bolsas) de 1 a 24 meses de edad con diarrea de duración inferior a 72 horas, tres o más deposiciones líquidas en las 24 horas anteriores y signos de deshidratación moderada o grave. Se excluyeron los niños con diarrea sanguinolenta, signos de infección sistémica que necesitara tratamiento intravenoso con antibióticos o malnutrición grave.

En el estudio participaron 675 niños: 341 que recibieron la SRO de osmolaridad reducida y 334 que recibieron la SRO tradicional de la OMS. La cantidad de heces (medida en gramos por kilogramo de peso) fue comparable con los dos tratamientos, tanto en las primeras 24 horas $(114 \mathrm{~g} / \mathrm{kg}$ con la SRO de osmolaridad reducida y $125 \mathrm{~g} / \mathrm{kg}$ con la SRO tradicional) como durante la totalidad del estudio (320 frente a $331 \mathrm{~g} / \mathrm{kg}$, respectivamente). La proporción de niños con vómitos durante las primeras 24 horas también fue similar con ambas soluciones $(58 \%$ con la SRO de osmolaridad reducida, frente a $62 \%$ con la SRO tradicional). Lo mismo ocurrió con la duración de la diarrea. En cambio, la proporción de niños que necesitaron tratamiento intravenoso no programado fue significativamente menor en los tratados con la SRO de osmolaridad reducida $(10 \%)$ que en los tratados con la SRO tradicional $(15 \%)$. No hubo diferencias significativas entre los dos grupos con respecto a la incidencia de hiponatremia (sodio sérico $<130 \mathrm{mmol} / \mathrm{L}$ ) a las 24 horas $(11 \%$ con la SRO de osmolaridad reducida y $9 \%$ con la SRO tradicional).

De acuerdo con estos resultados, la SRO de osmolaridad reducida redujo la necesidad de tratamiento intravenoso no programado en un 33\% y no tuvo efectos detectables sobre la cantidad de heces ni la duración de la diarrea. Esto indica que la SRO de osmolaridad reducida puede ser beneficiosa en niños con diarrea aguda, pero antes de llegar a un consenso sobre la composición de la SRO también deberían tenerse en cuenta los resultados de los ensayos clínicos que investiguen la eficacia y seguridad de la SRO de osmolaridad reducida en adultos con cólera. (CHOICE Study Group. Multicenter, randomized, double-blind clinical trial to evaluate the efficacy and safety of a reduced osmolarity oral rehydration salts solution in children with acute watery diarrhea. Pediatrics 2001;107:613-618) 